\title{
Infrared Reflection-Absorption Spectra of Calcium Icosanate Langmuir-Blodgett Films Observed by Using Zinc Oxide-Based Transparent Electrode and Gold
}

\author{
Takashi Sugimoto, Keiichi Ikegami*, Takashi Yoshiyama ${ }^{1)}$, Keigou Maejima ${ }^{1)}$, \\ Hajime Shibata $^{2)}$, Hitoshi Tampo ${ }^{1)}$, and Shigeru Niki ${ }^{1)}$ \\ Nanotechnology Research Institute, National Institute of Advanced Industrial Science and Technology (AIST) \\ AIST Tsukuba Central-2, 1-1-1 Umezono, Tsukuba 305-8568, Japan \\ ${ }^{1)}$ Research Center for Photovoltaics, AIST, 1-1-1 Umezono, Tsukuba 305-8568, JAPAN \\ 2) Nanoelectronics Research Institute, AIST, 1-1-1 Umezono, Tsukuba 305-8568, JAPAN \\ Fax: 81-29-861-5400, e-mail: k.ikegami@aist.go.jp
}

\begin{abstract}
As a method for characterizing the electronic state and molecular orientation of organic materials put on electrodes, infrared reflection-absorption spectroscopy (IR-RAS) is a well-known powerful tool. We have demonstrated that transparent electrodes based on zinc oxide are useful as substrates for the IR-RAS measurements. Although their absorption-signal enhancement effect is smaller than that of gold substrates, sometimes IR-RAS with them provides a better basis than that with gold for discussing the molecular orientation.
\end{abstract}

Key words: Reflection-absorption spectroscopy, transparent electrode, IR absorption, Langmuir-Blodgett film

\section{INTRODUCTION}

Zinc Oxide-based transparent electrodes $[1,2]$ have been attracting increasing attention because of the sparsity of indium, which is the major constituent of the present industrially standard transparent conducting material, ITO (indium-tin oxide). In the future, $\mathrm{ZnO}$-based transparent electrodes will be widely used in optoelectronic industries, including those utilizing organic materials. However, characterization of the electronic and orientation states of the molecules or molecular groups deposited upon them has not yet been focused.

As a method for characterizing the electronic state and molecular orientation of organic materials put on electrodes, infrared reflection-absorption spectroscopy (IR-RAS) is a well-known powerful tool. For example, mono- and multilayered Langmuir-Blodgett (LB) films of cadmium octadecanate were intensively studied by this method with silver substrates [3].

The high sensitivity of the IR-RAS method originates from enhancement of the optical electric field induced by the plasma oscillation of the free carriers in the metal substrates used. Therefore, since the plasma frequency of a transparent electrode is larger than the mid-infrared frequency [4], it is expected that the materials deposited upon transparent electrodes can be characterized by means of IR-RAS. Actually, on an ITO electrode, Paniagua et al. [5] succeeded to observe IR-RAS signal of phosphonic acids. However, in their case, the photoelastic modulation method was used to enhance the signal-to-noise ratio, and, therefore, their results do not assure the effectiveness of the simple IR-RAS method with transparent electrodes.
More recently, we demonstrated that the simple IR-RAS method is effective in characterizing organic materials deposited on a transparent electrode based on Ga-doped zinc oxide covered with a buffer layer, even when the quantity of the material is at the monolayer level [6]. Namely, the correlation between formation of the J-aggregate and changes in electronic structure of the dye chromophore was clearly shown by comparing the usual transmission visible spectra and IR-RAS signals of a merocyanine-dye LB films deposited with and without a divalent metal cation. It should be emphasized that, in this dye LB film case, the transparency of the substrate in the visible region was another crucial key. This transparency may help researcherss in various situations. However, the reported results were insufficient to estimate to what extent the $\mathrm{ZnO}$-based transparent electrode is useful as a substrate for the IR-RAS measurements because control experiments with metal substrates were not executed.

In this work, therefore, we intended to compare the intensities of IR-RAS signals of LB films deposited on a $\mathrm{ZnO}$-based transparent electrode and gold to each other. In addition, this time we chose $\mathrm{Al}$-doped $\mathrm{ZnO}$ without a buffer layer to see that dopant species and the insertion of a buffer layer are not crucial. The results presented in this paper confirm that the ZnO-based transparent electrode is useful for the IR measurements, though its absorption-signal enhancement effect is smaller than that of gold. Furthermore, the fact that the materials deposited on these substrates can be in electronically and structurally different states imply that the more appropriate substrate for the IR-RAS measurements should be selected depending on the aim. 


\section{EXPERIMENTAL}

An aluminum-doped zinc oxide (hereafter abbreviated as AZO) films was deposited onto $10 \times 10 \mathrm{~cm}$ glass plates by $\mathrm{rf}$ magnetron sputtering, as in the case of the previous works [4]. Its thickness and conductivity, which were measured by a surface profiler and the van der Pauw method, were $518 \mathrm{~nm}$ and $2.67 \mathrm{~S} / \mathrm{cm}$, respectively. Its plasma frequency was estimated at $2.7 \times 10^{15} \mathrm{~s}^{-1}$ (corresponding to a wavelength of $700 \mathrm{~nm}$ ) based on the optical measurements in the previous study [4]. The AZO substrates were prepared by cutting the AZO-coated glass into pieces with the dimensions of 3.8 $\times 1.3 \mathrm{~cm}$. Gold substrates were prepared by evaporating gold onto chromium-coated glass with the same dimensions. The thicknesses of the $\mathrm{Au}$ and $\mathrm{Cr}$ layers are 100 and $5 \mathrm{~nm}$, respectively.

A chloroform solution $\left(1.55 \times 10^{-3} \mathrm{~mol} / \mathrm{L}\right)$ of fully deuterated icosanoic acid, $\mathrm{C}_{20}-\mathrm{d}_{39}(\mathrm{H})$, which was purchased from $\mathrm{CDN}$ isotopes, Inc., was spread upon the surface of a $\mathrm{CaCl}_{2}$ aqueous solution $\left(1 \times 10^{-3} \mathrm{~mol} / \mathrm{L}\right.$, including $1 \times 10^{-4} \mathrm{~mol} / \mathrm{L}$ of $\left.\mathrm{K}_{2} \mathrm{CO}_{3}, \mathrm{pH}=6.3\right)$ controlled at $20{ }^{\circ} \mathrm{C}$ to prepare Langmuir films. These Langmuir films were compressed at the surface pressure of 40 $\mathrm{mN} / \mathrm{m}$ and then transferred onto the substrates as LB films by the vertical dipping method. The withdrawing speed of the substrate for the first layer deposition was $1.5 \times 10^{-3} \mathrm{~cm} / \mathrm{s}$. Afterward, the immersing and withdrawing speeds of the substrates were set to $4.0 \times$
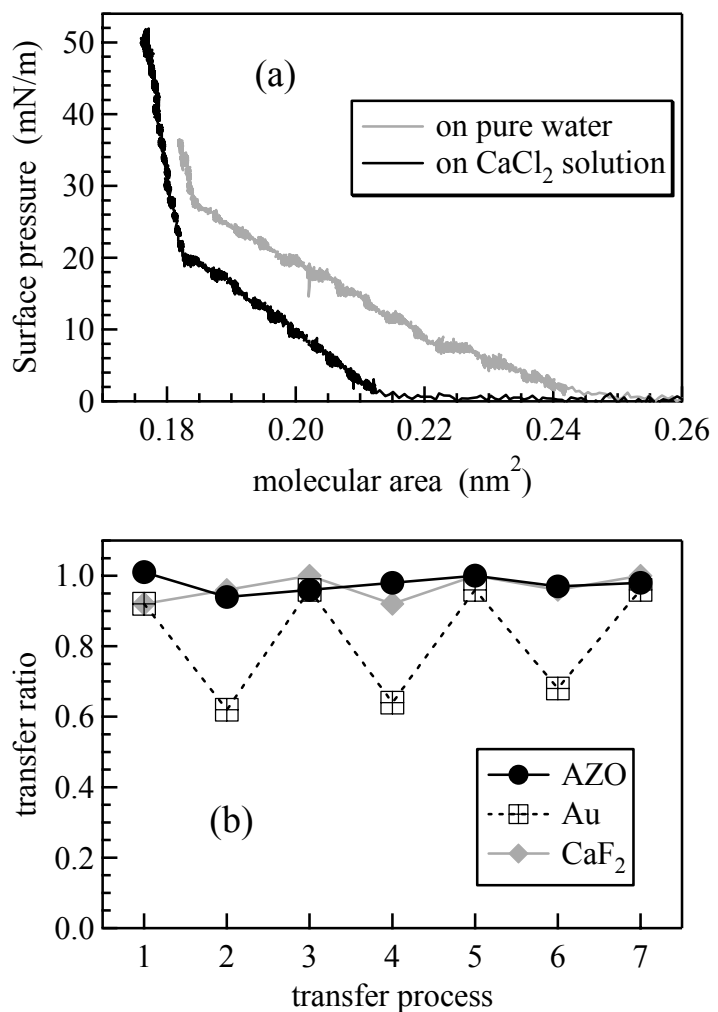

Fig. 1 (a) Surface pressure-molecular area isotherm observed for $\mathrm{C}_{20}-\mathrm{d}_{39}$ spread upon a $\mathrm{CaCl}_{2}$ aqueous solution measured by the step-by-step method. That observed on pure water is also indicated for comparison. (b) Transfer ratio in each withdrawing (even numbered) and immersing (odd-numbered) process.
$10^{-2} \mathrm{~cm} / \mathrm{s}$ and $7.5 \times 10^{-3} \mathrm{~cm} / \mathrm{s}$, respectively. Pure water with a resistivity higher than $1.8 \times 10^{7} \Omega \mathrm{cm}$ from a Millipore Milli-Q system and a Lauda Filmwaage Langmuir trough were used.

IR-RAS measurements were performed by a Perkin-Elmer Spectrum-2000 spectrometer with a Refractor-2 attachment from Harrick Scientific Products, Inc., in which the angle of incidence is set to $75^{\circ}$.
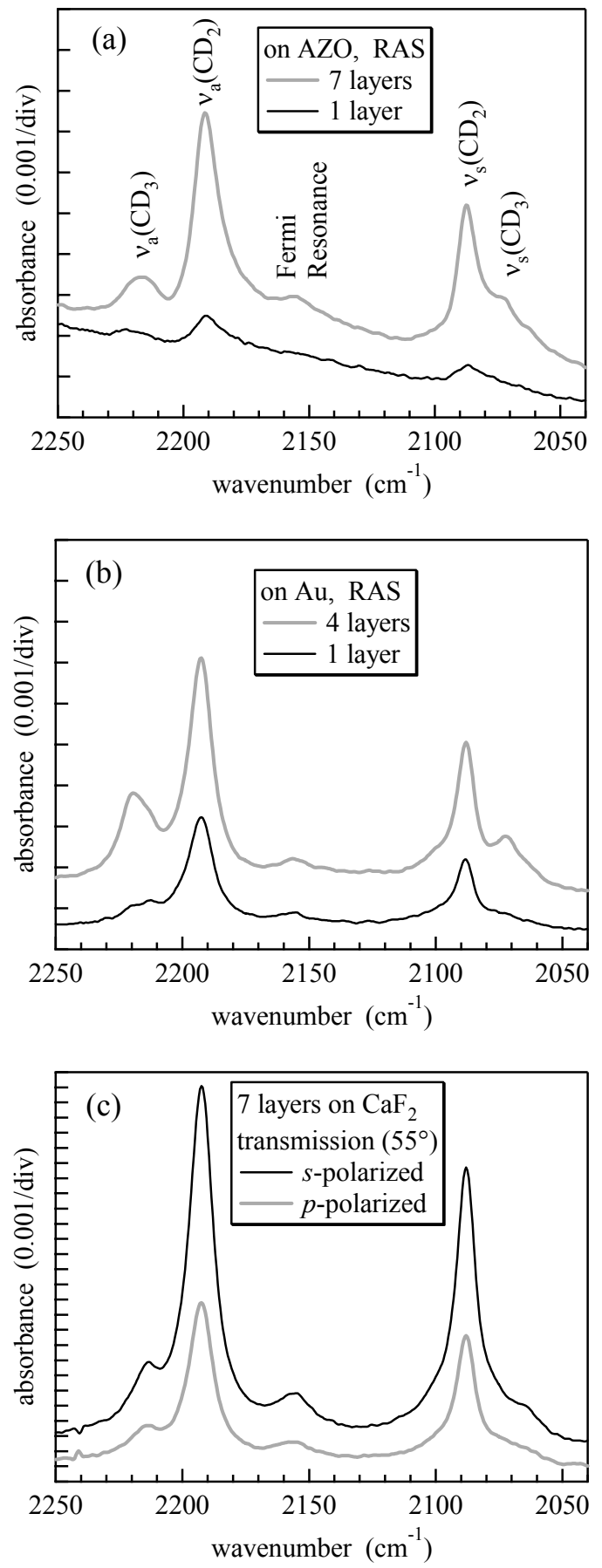

Fig. 2 IR absorption spectra in the $\mathrm{C}-\mathrm{D}$ stretching region observed for $\mathrm{C}_{20}-\mathrm{d}_{39}(\mathrm{Ca}, \mathrm{H}) \mathrm{LB}$ films deposited on (a) $\mathrm{AZO}$, (b) $\mathrm{Au}$, and (c) $\mathrm{CaF}_{2}$ substrates. 


\section{RESULTS AND DISCUSSION}

3.1 $F-A$ isotherm and LB transfer Surface pressure-molecular area $(F-A)$ isotherm of $\mathrm{C}_{20}-\mathrm{d}_{39}$ spread upon the $\mathrm{CaCl}_{2}$ aqueous solution (Fig. 1 (a)) was measured by the so-called step-by-step method [7]. (In this method, the negative-feedback routine in the trough controller is switched on and the target surface pressure is increased with $2 \mathrm{mN} / \mathrm{m}$ steps. At each step, the films are allowed to relax for sufficient time, i.e., the stabilization of the area is waited for before increasing the target surface pressure for the next step.) $\quad \mathrm{C}_{20}-\mathrm{d}_{39}$ spread upon the Ca-containing subphase shows a limited occupied area of approx. 0.21 $\mathrm{nm}^{2}$, being smaller than that of $\mathrm{C}_{20}-\mathrm{d}_{39}$ on a pure-water subphase. However, the observed gradual increase in $F$ below $F=20 \mathrm{mN} / \mathrm{m}$ and the phase transition around that $F$ suggest that the formed Langmuir film contains unionized acid. Thus, hereafter we abbreviate the present material as $\mathrm{C}_{20}-\mathrm{d}_{39}(\mathrm{Ca}, \mathrm{H})$. Nevertheless, the film is stable till the surface pressure is increased to $52 \mathrm{mN} / \mathrm{m}$. Under this surface pressure, the molecular area is approx. $0.18 \mathrm{~nm}^{2}$, implying that the alkyl chains are closely packed. We have selected this mixed material for a testing sample, since it provides several absorption bands related to the carboxylic group and then convenient for checking the ability of AZO as a substrate for the IR-RAS measurements.

LB transfer of $\mathrm{C}_{20}-\mathrm{d}_{39}(\mathrm{Ca}, \mathrm{H})$ onto the bare AZO substrates is nearly Y-type (Fig. 1 (b)), as in the case of $\mathrm{CaF}_{2}$ and glass substrates. On the contrary, based on the data in Fig. 1 (b), the transfer of the material on to the $\mathrm{Au}$ substrates is recognized as nearly Z-type, since the rear side of the substrate is glass, on which the transfer ratio should be close to unity. (Note that the rear side of the AZO substrate is also glass.) Then, the immersing process should not be taken into account in countering the layer number in the Au-substrate case, i.e., the sample prepared by 4 withdrawing and 3 immersing processes is considered as a 4 layered sample.

\subsection{IR-RAS signal intensity}

Our major concern is focused on the extent to which the $\mathrm{ZnO}$-based transparent electrode is useful as a substrate for the IR-RAS. For seeing this, the signal intensity of the absorption bands due to the C-D stretching modes may be a good indicator because it is
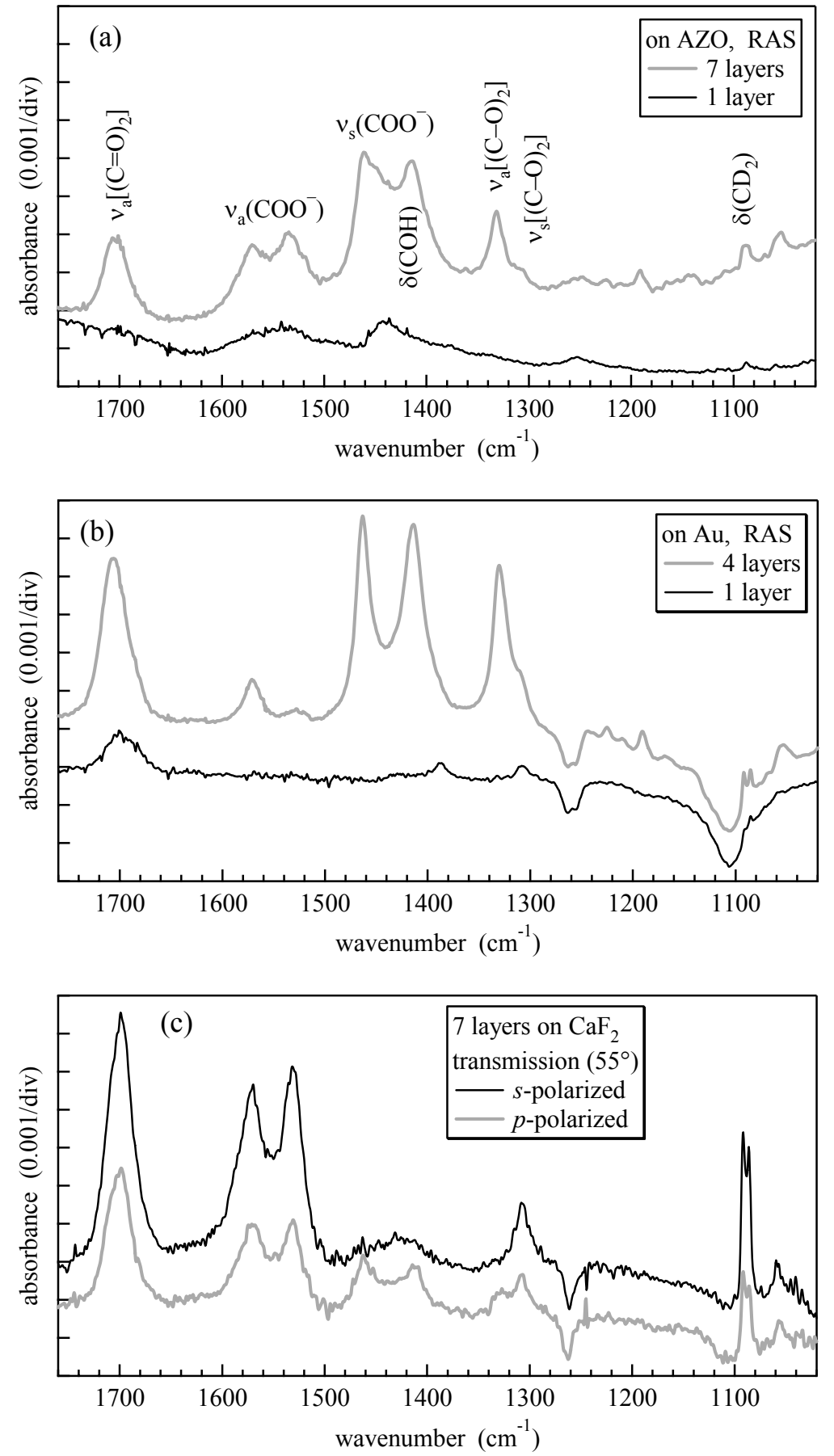

Fig. 3 IR absorption spectra in the "fingerprint" region observed for $\mathrm{C}_{20}-\mathrm{d}_{39}(\mathrm{Ca}, \mathrm{H}) \mathrm{LB}$ films deposited on (a) AZO, (b) $\mathrm{Au}$, and (c) $\mathrm{CaF}_{2}$ substrates. The $v_{\mathrm{s}}\left(\mathrm{COO}^{-}\right)$and $\mathrm{COH}$ deformation modes are overlapped with each other..

expected to be sufficiently strong and well isolated from background signals caused by water vapor and oily pollutants. At the first glance, it is noted that the $v_{\mathrm{a}}\left(\mathrm{CD}_{2}\right)$ and $v_{\mathrm{s}}\left(\mathrm{CD}_{2}\right)$ bands can be clearly detected by the IR-RAS method performed with the AZO substrate (Fig. 2 (a)), even in the 1-layer case. Moreover, some vibronic absorption bands assignable to the carboxylic group are clearly seen even for the 1-layer sample (Fig. 3 (a)). These results are consistent with the reported results that 
the aggregating state of a merocyanine dye in a monolayer-like system deposited on Ga-doped $\mathrm{ZnO}$ substrates could be investigated by the IR-RAS method [6].

Quantitative comparison with the IR-RAS on the Au substrates should be done for thicker samples. Comparing the $v_{\mathrm{a}}\left(\mathrm{CD}_{2}\right)$ and $v_{\mathrm{s}}\left(\mathrm{CD}_{2}\right)$ bands displayed in Fig. 2 (a) to those in Fig. 2 (b), the absorption-signal enhancement effect of the AZO substrate is roughly estimated at $1 / 2$ of that of the $\mathrm{Au}$ substrate. Here, however, we must pay attention to possible difference in the orientation of the alkyl chains in these two cases. Comparing the intensities of the bands in the "fingerprint" region (Fig. 3 (a) and (b)) may indicate that the enhancement effect of the AZO substrate is larger than $1 / 4$ of that of the Au substrate. Thus, we can conclude that the $\mathrm{ZnO}$-based transparent electrodes are useful in performing the IR-RAS measurements, regardless of the dopant species and insertion of a buffer layer.

\subsection{Molecular orientation}

Under the RAS conditions, the optical electric field in the sample material is normal to the substrate surface. Besides the signal enhancement effect, this orientation of the optical electric field is another advantage of the RAS method, because it can be quite helpful in evaluating the molecular orientation in the organized molecular films such as LB films. That is, comparison of the absorption spectra observed with the RAS and transmission method, in the latter of which the optical electric field is parallel to the substrate surface (with the $s$-polarization) or oblique (with the $p$-polarization), provides information about the orientation of the transition dipole moments [3]. However, this comparison may be useless if the structure of the films deposited on the substrates for the RAS and transmission methods are largely different.

Actually, the $\mathrm{C}_{20}-\mathrm{d}_{39}(\mathrm{Ca}, \mathrm{H}) \mathrm{LB}$ films deposited on the $\mathrm{Au}$ substrate is Z-type as pointed in Sec. 3.1, while those on the $\mathrm{CaF}_{2}$ substrate is Y-type. This difference in the layered structure may cause difference in the molecular orientation. On the contrary, the $\mathrm{C}_{20}-\mathrm{d}_{39}(\mathrm{Ca}, \mathrm{H}) \mathrm{LB}$ films deposited on the AZO substrate is Y-type. Therefore, to discuss the molecular orientation, the IR-RAS spectrum of the 7-layered film on the AZO substrate is more useful than that of the 4-layered film on the Au substrate.

IR absorption spectra observed for a 7-layered LB films on the $\mathrm{CaF}_{2}$ substrate by the oblique transmission method (the angle of incidence set to $55^{\circ}$ ) with the s- and p-polarization are indicated in Figs. 2 (c) and 3 (c). Comparing these to the corresponding one in Figs. 2 (a) and 3 (a), the followings are deduced. i) The alkyl chains are preferentially oriented normal to the substrate surface, but the tilting angle is not very small. ii) The acidic carboxylic group in the film forms a ring dimer via hydrogen bonds. The transition dipole moment of the $v_{\mathrm{a}}\left[(\mathrm{C}=\mathrm{O})_{2}\right]$ mode, which is parallel to the plane of the ring dimer and perpendicular to the $\mathrm{C}=\mathrm{O}$ bond, is preferentially parallel to the substrate surface. iii) The moments of the $v_{\mathrm{a}}\left[(\mathrm{C}-\mathrm{O})_{2}\right]$ and $v_{\mathrm{s}}\left[(\mathrm{C}-\mathrm{O})_{2}\right]$ modes are nearly normal and parallel to the substrate surface, respectively. iv) The films contain two kinds of the anionic carboxylic group, bringing about the splits of the $v_{\mathrm{a}}\left(\mathrm{COO}^{-}\right)$and $v_{\mathrm{s}}\left(\mathrm{COO}^{-}\right)$modes. However, the transition dipole moments of the both $v_{\mathrm{a}}\left(\mathrm{COO}^{-}\right)$bands, which are parallel to the line connecting two $\mathrm{O}$ atoms, have the same orientation: preferentially parallel to the substrate surface. v) The transition dipole moments of the both $v_{\mathrm{s}}\left(\mathrm{COO}^{-}\right)$bands, which are parallel to the symmetrical line of the $\mathrm{COO}$ triangle, have the same orientation: nearly normal to the substrate surface.

\section{CONCLUSION}

We have demonstrated that $\mathrm{ZnO}$-based transparent electrode is useful as a substrate for the IR-RAS measurements. In addition, when compared to $\mathrm{Au}$, at least in some cases, the structure of materials deposited on $\mathrm{ZnO}$-based transparent electrode is much more compatible with that on substrates for the transmission method. In such cases, IR-RAS measurements with ZnO-based transparent electrode provide a better basis to discuss the molecular orientation than those with $\mathrm{Au}$.

\section{REFERENCES}

[1] T. Yamamoto, T. Yamada, A. Miyake, H. Makino, and N. Yamamoto, J. Soc. Inf. Display, 16 713-719 (2008).

[2] T. Minami, Semicond. Sci. Technol., 20 S35-S44 (2005).

[3] J. Umemura, T. Kamata, T. Kawai, and T. Takenaka, J. Phys. Chem., 94 62-67 (1990).

[4] K. Ikegami, T. Yoshiyama, K. Maejima, H. Shibata, H. Tampo, S. Niki, J. Appl. Phys., 105 093713-1093713-7 (2009).

[5] S.A. Paniagua, P.H. Hotchkiss, S.C. Jones, S.R. Marder, A. Mudalige, F.S. Marrikar, J. E. Pemberton, N.R. Armstrong, J. Phys. Chem. C, 112 7809-7817 (2008).

[6] K. Ikegami, T. Yoshiyama, K. Maejima, H. Shibata, H. Tampo, S. Niki, Thin Solid Films 518 462-465 (2009).

[7] K. Ikegami, C. Mingotaud, P. Delhaès, Phys. Rev. E, 56 1987-1997 (1997).

(Received December 16, 2009; Accepted April 5, 2010) 